\title{
Pd nanowire coatings of laser-treated polyethylene naphthalate: Preparation, characterization and biological response
}

\author{
M. Polívková ${ }^{1}$, M. Valová ${ }^{1}$, S. Rimpelová ${ }^{2}$, P. Slepička $^{1}$, V. Švorčík ${ }^{1}, J$. Siegel ${ }^{1}$ \\ ${ }^{1}$ Department of Solid State Engineering, University of Chemistry and Technology Prague, 16628 Prague, Czech Republic \\ ${ }^{2}$ Department of Biochemistry and Microbiology, University of Chemistry and Technology Prague, 16628 Prague, Czech \\ Republic
}

Received 27 April 2018; accepted in revised form 20 June 2018

\begin{abstract}
Polymeric biomaterials treated by nanostructured metal coatings are very efficient against a wide spectrum of nosocomial pathogens. One of the most effective ways for the preparation of such metal/polymer composites is the combination of excimer laser modification of polymeric materials and vacuum evaporation of noble metals. By this way, we successfully prepared palladium nanowire arrays (PdNWs) supported on biocompatible polyethylene naphthalate (PEN). The characterization of prepared PdNWs on the surface of PEN was accomplished by various methods, such as X-ray Photoelectron Spectroscopy (XPS), Focussed Ion Beam Scanning Electron Microscopy (FIB-SEM), and Atomic Force Microscopy (AFM). PdNWs were preferentially formed from one side of underlying ripples. Pd release in antibacterial testing was measured by Inductively coupled plasma mass spectrometry (ICP-MS). Then, the antibacterial and cytotoxic effects were evaluated by (i) drop plate method using E. coli $\left(\mathrm{G}^{-}\right)$and $S$. epidermidis $\left(\mathrm{G}^{+}\right.$bacteria), and (ii) WST-1 cytotoxicity assay with three model cell lines (L929, NIH 3T3, RAW 264.7), respectively. Pd-treated samples exhibited significant antibacterial effects, increasing with cultivation time. Cytotoxicity assay showed that the absorbance of PEN/PdNWs samples was mildly decreased, suggesting considerably low cytotoxic effects of PdNWs.
\end{abstract}

Keywords: nanocomposites, coatings, laser patterning, antibacterial effects, cytotoxicity

\section{Introduction}

Nanostructured palladium is an important reagent in many industrial applications. Typically, it is widely used as a catalyst for the reduction of automotive pollutants [1], as well as in many organic reactions, such as Suzuki, Heck and Stille ones [2]. It is also significantly effective material for the detection of hydrogen in hydrogen-storage facilities [3]. All of these applications require the use of $\mathrm{Pd}$ in a finely ground state, where the size and shape are most important parameters maximizing the response of Pdbased elements. The ability to produce Pd nanostructures in various geometric shapes provides a great opportunity to systematically explore their electrical, catalytic and plasmon-resonance properties, as well as fully broaden their potential applications, especially in the field of polymeric medical devices with antibacterial response [4].

One of the most effective antibacterial treatments of biocompatible polymers represents nanostructured metal-based coatings, such as various types of $\mathrm{TiO}_{2}$ nanostructures $[5,6]$, because they often exhibit strong antibacterial efficacy without bacterial resistance. With the rapid development of nanotechnology, nanostructured metal coatings can be prepared by various techniques. Among the most important

${ }^{*}$ Corresponding author, e-mail: polivkoa@,vscht.cz (C) BME-PT 
ones are physical and chemical vapor depositions, chemical impregnation, lithographic methods, homogeneous reactions in a solution and electrodeposition [7].

Nowadays, nanostructured silver is the mostly used noble metal in medical applications. In recent days, however, cytotoxic studies warning about the toxicity of Ag nanostructures began to appear [8]. Some of these studies indicate that the most cytotoxic type of nanostructured silver materials are $\mathrm{Ag}$ nanowires $[9,10]$. For this reason, Pd nanowires (PdNWs) were studied in this work as a 'safer' alternative with potential to substitute $\mathrm{Ag}$ in medical applications. This, however, may be successfully accomplished under two conditions: (i) Pd exhibits antibacterial response comparable to Ag, while (ii) its cytotoxicity is significantly lower. Specifically, polyethylene naphthalate (PEN)/palladium nanowires composites (PEN/ PdNWs) were prepared by the combination of PEN modification by excimer laser followed by subsequent vacuum evaporation of $\mathrm{Pd}$. Metal evaporation proceeded under tilted angle geometry.

\section{Experimental}

\subsection{Materials, apparatus and procedures}

Polyethylene naphthalate foil (PEN, thickness $50 \mu \mathrm{m}$, density $1.36 \mathrm{~g} \cdot \mathrm{cm}^{-3}$ ) supplied by Goodfellow Ltd., was exposed to $\mathrm{KrF}$ excimer laser (COMPexPro $50 \mathrm{~F}$, Coherent, Inc.) with pulse duration of $20-40 \mathrm{~ns}$, frequency of $10 \mathrm{~Hz}$ and wavelength of $248 \mathrm{~nm}$. A laser beam was linearly polarized by fused quartz prism (model PBSO - 248-100) and directed through the aperture $\left(5 \times 10 \mathrm{~mm}^{2}\right)$ to the polymer. PEN was modified under static conditions with 6000 pulses and laser fluence of $10 \mathrm{~mJ} \cdot \mathrm{cm}^{-2}$ [10]. Controlled movement of the sample holder allowed multiple sample irradiation, producing a modified area of $2 \times 2 \mathrm{~m}^{2}$.

Pd nanowire arrays (thickness of $20 \mathrm{~nm}$, length of $20 \mathrm{~mm}$ ) were prepared on the surface of laser-modified PEN by vacuum evaporation (LEYBOLD-Heraeus, Univex 450). Pd was deposited using 99.99\% pure Pd pellets $\left(3.18 \times 3.18 \mathrm{~mm}^{2}\right.$, Safina, a.s., CZ). The evaporation process was performed at room temperature, total pressure of $3 \cdot 10^{-4} \mathrm{~Pa}$, and under a glancing angle of $70^{\circ}$. Quartz crystal microbalance was used for in situ monitoring of Pd thickness ( $\Delta f=$ $220 \mathrm{~Hz}$ for $20 \mathrm{~nm}$ Pd thickness) and verified by AFM scratch method (AFM VEECO CP II device, glass substrate).

\subsection{Analytical methods}

The atomic concentrations of $\mathrm{Pd}(3 \mathrm{~d}), \mathrm{C}(1 \mathrm{~s})$ and $\mathrm{O}(1 \mathrm{~s})$ were studied by X-ray photoelectron spectroscopy (XPS) on Omicron Nanotechnology ESCAProbeP spectrometer. The $\mathrm{X}$-ray source was monochromated at $1486.7 \mathrm{eV}$. The evaluation of spectra was performed by CasaXPS software. The samples were studied under the electron take-off angles of 0 and $\pm 81^{\circ}$.

Focused Ion Beam Scanning Electron Microscope (FIB-SEM, LYRA3 GMU, Tescan, Czech Republic) was used for visual representation of $\mathrm{Pd}$ nanowire arrays. FIB cuts were made by a Gallium ion beam. The polishing procedure was performed to clean and flatten the analyzed surface. The image was taken under the observation angle of $54.81^{\circ}$ and the voltage of $5 \mathrm{kV}$.

Surface morphology, roughness, periodicity and height were examined by AFM VEECO CP II device in a tapping mode. A Veeco oxide-sharpened P-doped silicon probe RTESPA-CP was used. Surface roughness, characterized by the mean roughness value $\left(R_{\mathrm{a}}\right)$, represents the arithmetic average of the deviation from the centre plane of a sample. Periodicity, characterizing how ripples/wires recur at intervals, was measured as a distance between two nearest bases/tops of ripples/wires. Height was measured from base to top of nanostructures. Variations of these parameters did not exceed 5\%.

Inductively coupled plasma with mass spectroscopy detector (ICP-MS) was used to determine the concentration of Pd released from the samples into physiological solution (PS, $0.9 \mathrm{wt} \% \mathrm{NaCl}$ ) under the same conditions as in antibacterial tests (see below). The trace element analysis of Pd leachates was performed using Agilent 8800 triple-quadrupole spectrometer (Agilent Technologies, Japan). The device was equipped by a collision cell (He collision gas) operating in a high-energy mode to minimize the interference of an analyte with $\mathrm{ArO}^{+}$adduct. Pure PS was used as a blank sample.

\subsection{Antibacterial tests}

Antibacterial response of the samples was investigated by the drop plate method in static $\left(24^{\circ} \mathrm{C}\right.$, laboratory table) and dynamic mode $\left(37^{\circ} \mathrm{C}\right.$, orbital shaker, $140 \mathrm{rpm}$ ) using two environmental bacterial strains; Gram-negative Escherichia coli (E. coli, DBM 3138) and Gram-positive Staphylococcus epidermidis (S. epidermidis, DBM 3179). Pure sterile PS 
$(2 \mathrm{~mL})$ served as a control. All experiments were accomplished under the sterile conditions. For experimental details see $[10,11]$.

\subsection{Cytotoxicity tests}

Cytotoxicity of the samples was examined using WST-1 assay (Sigma, USA) based on a conversion of tetrazolium salt into soluble formazan in viable cells, which is then spectrophotometrically measured. Three model cell lines were chosen: mouse embryonic fibroblasts L929 and NIH 3T3, and mouse macrophages RAW 264.7. Before this testing, the samples were sterilized by UV irradiation. Cells were also cultivated on standard tissue culture polystyrene (TCPS) for control. For details see ref. [12].

\section{Results and discussion}

\subsection{Preparation and surface characterization}

For the vacuum evaporation of $\mathrm{Pd}$, laser-pretreated (rippled) PEN was used as a template. Thereafter, vacuum evaporation process was carried out under the experimental set-up reported in [10] for AgNWs. Since this study aims to prepare antibacterial coatings with considerably lower cytotoxicity compared to commonly used AgNWs [10], the same nanowire thickness $(20 \mathrm{~nm})$ and other experimental conditions were used. These conditions enable the formation of fairly separated PdNWs, due to preferential Pd condensation. The fine structure of ripples is related directly to deposition at the glancing angle of incidence, being known as a shadow effect [13]. Under this phenomenon the spatial distribution of Pd flux is modulated, causing metal nucleation preferentially from one side of the ripples, near to the top of the illuminated ridges, where the flux is the highest. With the increasing Pd thickness, the agglomeration and coarsening of the metal clusters increases until a polycrystalline array of PdNWs is formed [14].

Preferential condensation of PdNWs was verified by visual representation of $\mathrm{Pd}$ nanowire arrays using FIB-SEM imaging (see Figure 1). One can observe the formation of ripples/wires uniformly distributed over the surface of polymer, which is in good accordance with subsequent AFM analysis (see Figure 2). SEM image showed the formation of malfunctions, producing visible wire twinning. Those deviations are, however, quite rare over the entire modified surface area. As available literature [15] confirms, forklike nanowire twinning is quite common phenomenon accompanying laser processing of polymeric materials, however, the reason for their formation is not yet precisely elucidated. Overall, FIB-SEM analysis is much more informative concerning the evaluation of nanowire shape and their distribution compared to AFM (see the text below); AFM technique is unable to visualize separated metal wires. Using FIB cuts, one can not only observe the position of metal on the modulated sample surface, but pointing

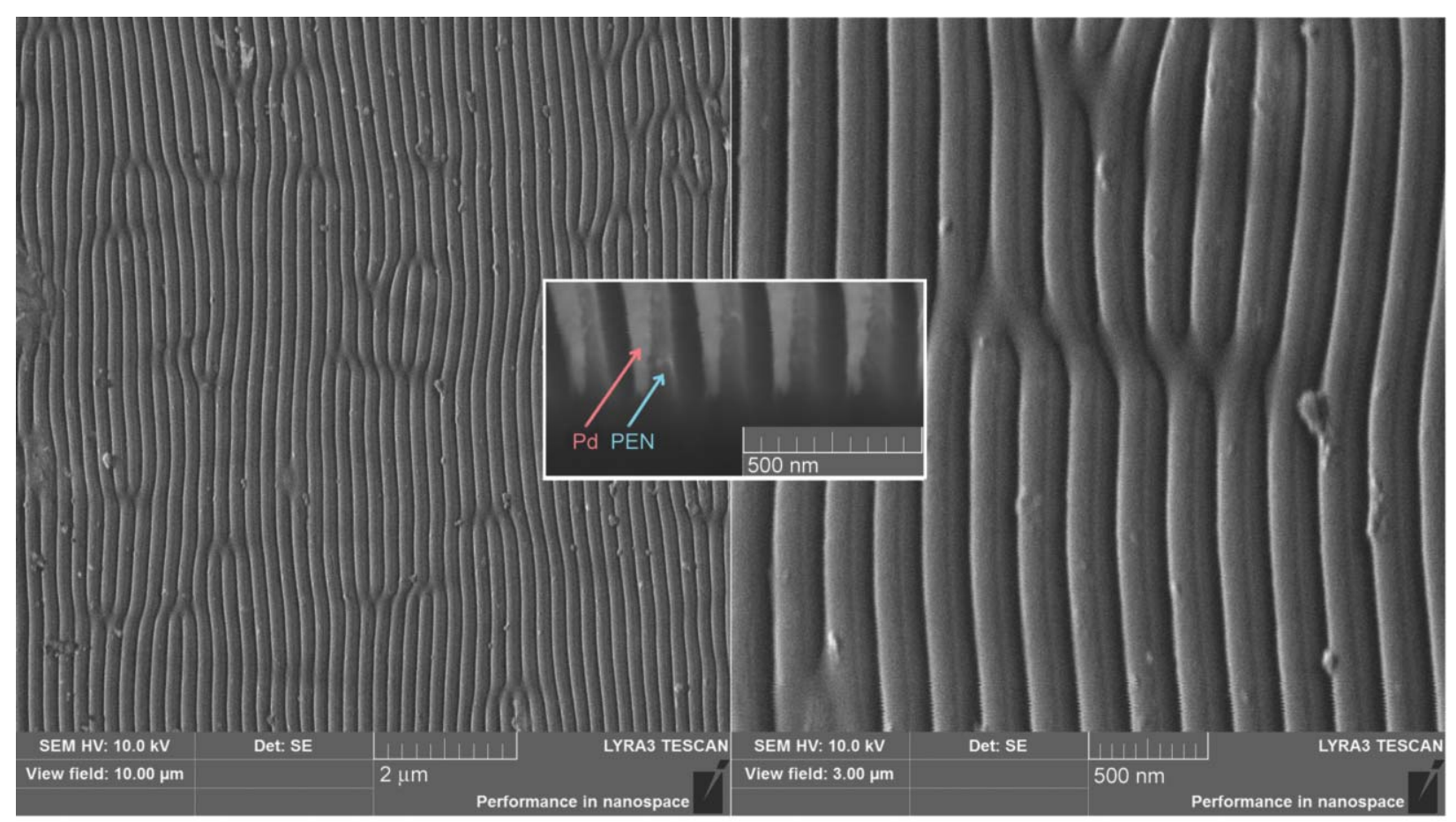

Figure 1. SEM image of PEN/PdNWs surface. The inset shows FIB-SEM cut of corresponding sample area. 
to analysis of the samples with different nanowire thickness, it is possible to distinguish morphological differences between individual nanowires [16]. In particular, FIB-SEM cut (Figure 1) confirmed the formation of $\mathrm{Pd}$ nanowires preferentially on directly exposed side (to the metal vapour flow) of the ripples, which corresponds to experimental set-up during the evaporation process.

Another way to verify the formation of Pd nanowires from one side of underlying ripples, is the use of
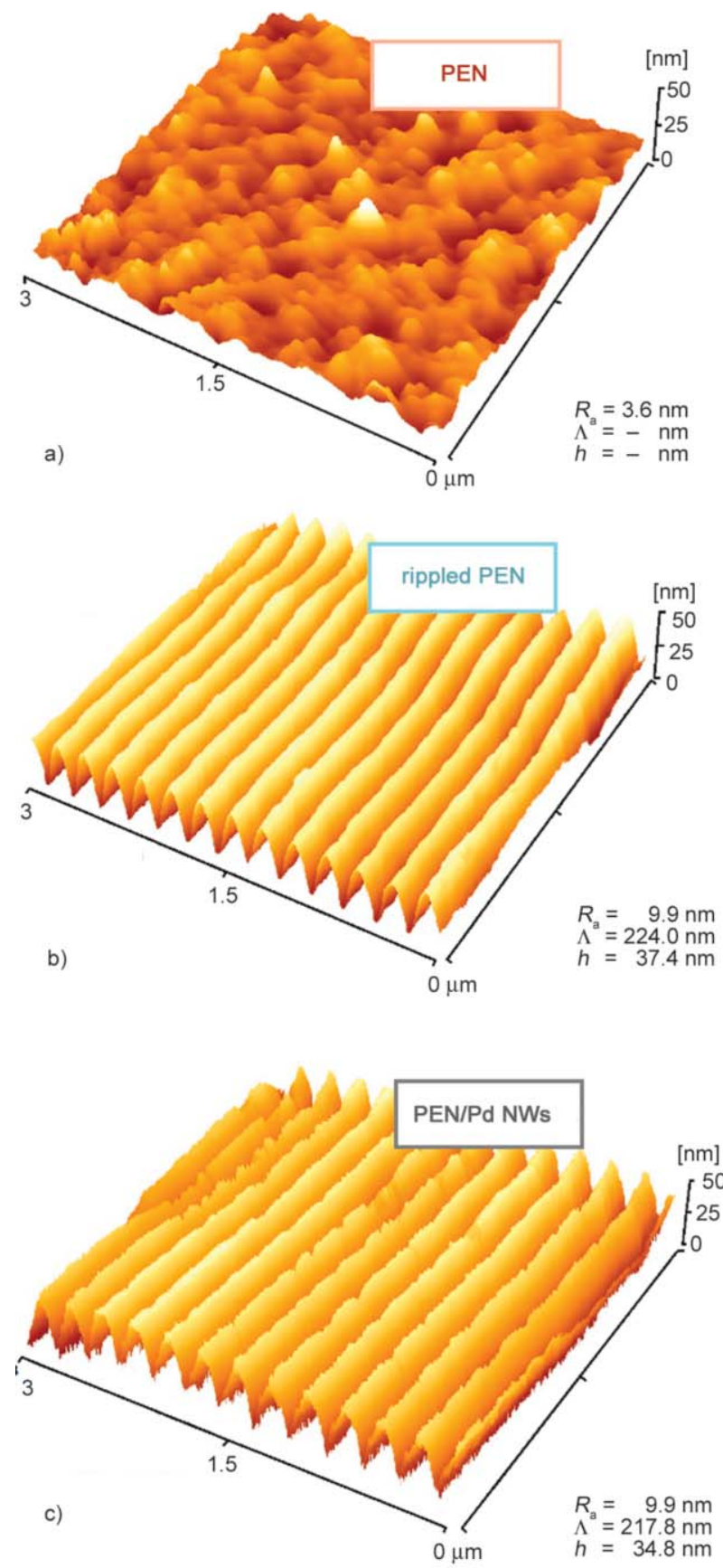

Figure 2. 3D AFM images of a) pristine, b) rippled PEN and c) PEN/PdNWs samples, together with corresponding values of surface roughness $\left(R_{\mathrm{a}}\right)$, periodicity $(\Lambda)$ and height $(h)$.
XPS analysis. Figure 3 summarizes the results of atomic concentrations of elements for pristine, rippled PEN and PEN/PdNWs samples. To clarify the direction of PdNWs formation, concentrations of elements were measured under the electron take-off angle of 0 , and $\pm 81^{\circ}$ from the left $(+)$ and right (-) side of the samples. The occurrence of $\mathrm{C}$ and $\mathrm{O}$ is given by PEN stoichiometry, and might be extended by the hydrocarbon impurities, which are often adsorbed on the surfaces of the polar polymeric materials from air [17]. Detected Pd originates from the evaporation process; formation of Pd NW arrays. The results showed that PEN/Pd samples measured under the electron take-off angle $81^{\circ}$ exhibited significant differences in the concentrations of Pd from $(+)$ and $(-)$ direction, which indicated the formation of PdNWs preferentially from one side of underlying ripples. The differences between the samples of pristine and laser-patterned PEN, particularly evident for the electron take-off angle of $81^{\circ}$, in case of both directions, were given by the change in the chemical composition caused by the exposure to laser beam, which was associated with the change in the surface morphology (see Figure 2a and 2b, AFM).

The surface morphology of the samples, characterized by $3 \mathrm{D}$ AFM scans together with the values of surface roughness $\left(R_{\mathrm{a}}\right)$, periodicity $(\Lambda)$ and height $(h)$ are shown in Figure 2. From AFM scans it is obvious that laser modification of pristine PEN (Figure 2a) led to the formation of periodic structures (ripples) uniformly distributed over the surface of PEN (Figure $2 b$ ). In particular, the modification of surface

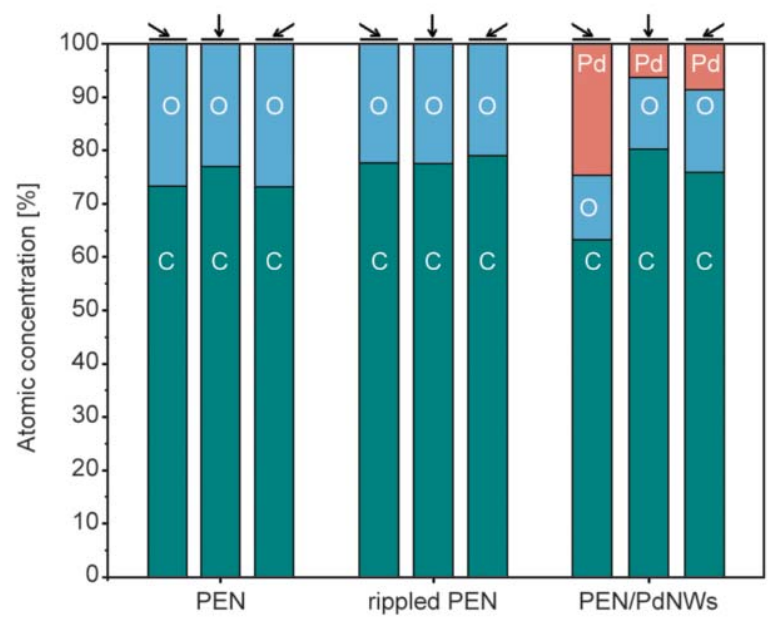

Figure 3. Atomic concentrations of $\mathrm{C}(1 \mathrm{~s}), \mathrm{O}(1 \mathrm{~s})$ and $\mathrm{Pd}(3 \mathrm{~d})$ measured at pristine, rippled PEN and PEN/PdNWs samples by XPS under the electron take-off angles of 0 and $\pm 81^{\circ}$, which is schematically visualized by appropriate arrows. 
morphology of polymers during laser patterning induces the variations of the values of surface roughness, as described elsewhere [16, 18]. Laser fluence and number of pulses have a major impact on the change of the surface roughness and morphology. The value of Ra for rippled PEN (Figure 2b) was approximately two-thirds higher than that one of pristine PEN (Figure 2a). Surprisingly, the same $R_{\mathrm{a}}$ was evaluated for the sample with deposited PdNWs (Figure 2c), while one can observe lower values of $\Lambda$ and $\mathrm{h}$ for PdNWs/PEN sample compared to rippled PEN (Figure b). This phenomenon is due to partial filling of ripples by metal during $\mathrm{Pd}$ deposition.

\subsection{Biological response}

$\mathrm{Pd}$ release into physiological solution after 3 and $24 \mathrm{~h}$ of static and dynamic leaching was studied by ICPMS. This measurement was used to outline the role of released Pd in antibacterial effects of the samples. Figure 4 shows the dependence of the concentrations of released $\mathrm{Pd}$ on time and mode of leaching for PEN/PdNWs samples. The concentration of Pd in pure physiological solution, pristine and rippled

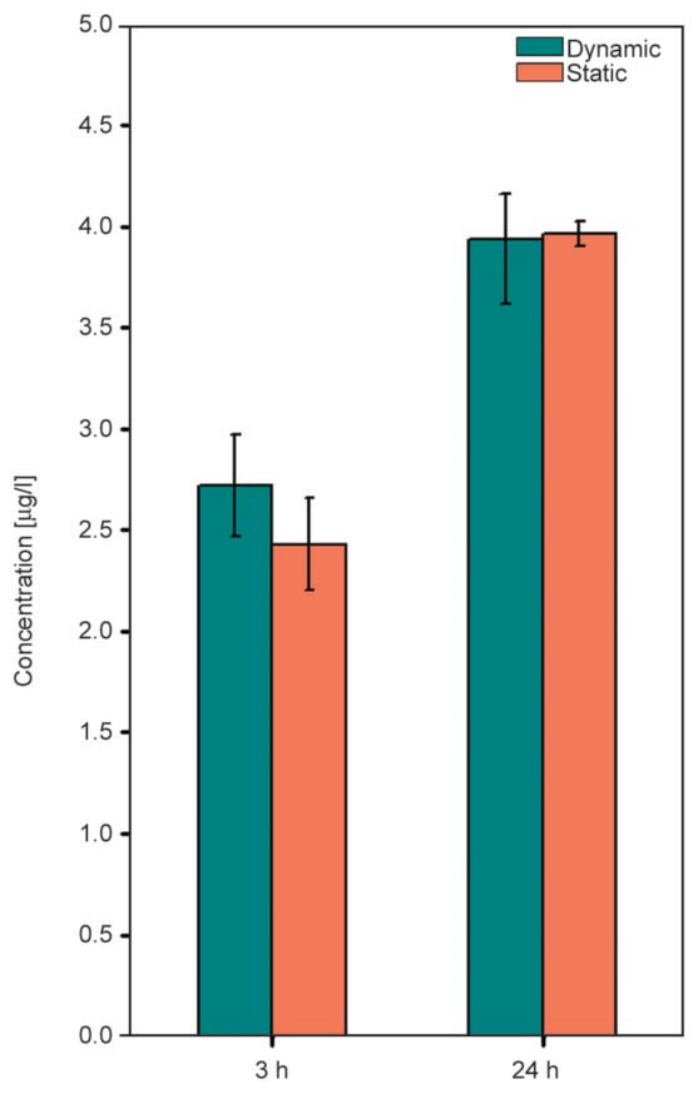

Figure 4. Concentration of $\mathrm{Pd}\left[\mu \mathrm{g} \cdot \mathrm{L}^{-1}\right]$ in dynamic and static leachates after 3 and $24 \mathrm{~h}$ of sample treatment measured by ICP-MS.
PEN samples was immeasurable. Regardless of the specific mode used (static and dynamic conditions), comparable amount of Pd was released. Similar trend was observed in case of both, Pd nanolayers and nanoisland, deposited on PEN [19], however, for Ag nanowires [10] prepared under identical conditions, the correlation between the used mode and Ag concentration was obvious; higher concentrations in dynamic shaking were detected. More specifically, Figure 4 revealed only the trend in increasing Pd concentration with time. Provided the Pd released plays the major role in antibacterial response of the samples, one can assume increased antibacterial effects of Pd samples at longer incubation time.

Antibacterial efficacy of PEN/PdNWs samples was studied by static and dynamic drop plate method using E. coli and S. epidermidis. Both bacteria were in direct contact with the tested polymer in PS and number of CFU was investigated after 3 and $24 \mathrm{~h}$ of cultivation. The results for pristine, rippled PEN, and PEN/PdNWs samples are summarized in Figure 5. One can observe generally higher amount of CFU in case of dynamic testing, which is in accordance with the dynamic mode conditions (140 rpm), which corresponds with standardly used conditions for the cultivation of inocula [20]. Pristine and rippled PEN were not antibacterially active under all conditions. In case of both strains and modes, PEN/PdNWs samples exhibited higher antibacterial effect after $24 \mathrm{~h}$ of cultivation. It corresponds with increasing concentration of released Pd with time (Figure 4 ICP-MS), as well as with longer contact of bacteria with the samples. Antibacterial effect of PEN/PdNWs was most pronounced in dynamic testing than in static one, in case of both bacterial strains. Because the concentrations of released Pd were comparable in both modes, observed increase in antibacterial response was predominantly caused by a different contact of bacteria with the samples (static immersion vs dynamic shaking). Generally higher antibacterial response against $S$. epidermidis might be explained by different composition of cell walls of $\mathrm{G}^{-}$and $\mathrm{G}^{+}$ bacteria; positively charged particles of noble metals penetrate more facile through the cell walls of $\mathrm{G}^{+}$ bacteria (S. epidermidis) [21]. Under identical conditions of antibacterial testing, PdNWs showed comparable antibacterial effects to AgNWs of the same thickness [10]. This finding could have opened the way for alternative applications of PdNWs instead of commonly used $\mathrm{Ag}$ ones in medical applications 

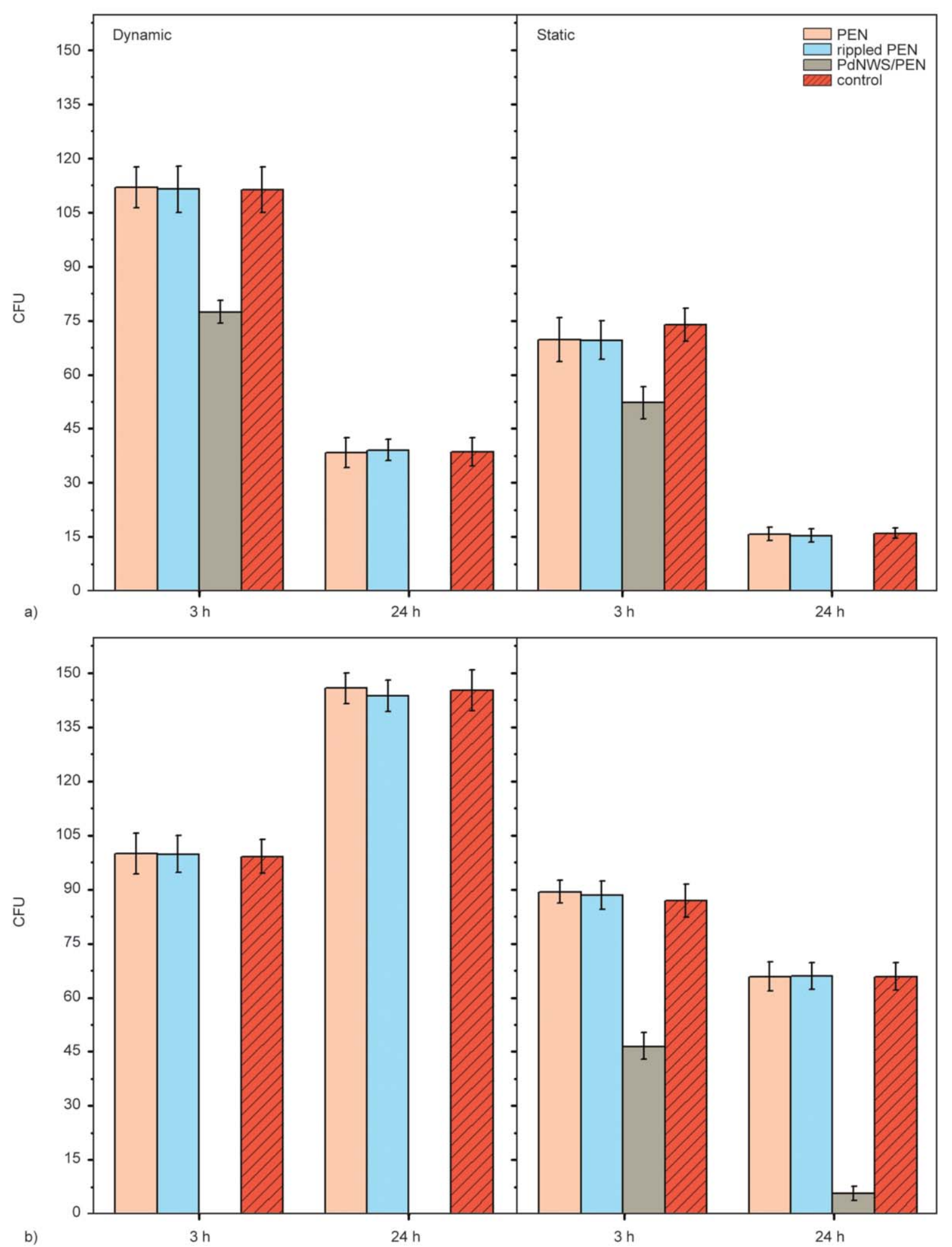

Figure 5. CFU of E. coli (a) and S. epidermidis (b) incubated with pristine, rippled PEN and PEN/PdNWs samples for 3 and $24 \mathrm{~h}$.

(antibacterial coatings of catheters and other medical devices [22]).

Cytotoxicity of PEN/PdNWs samples was studied using WST-1 assay after 24, 48 and $72 \mathrm{~h}$ of incubation on three model cell lines (L929, NIH 3T3 and RAW 264.7). The results were compared with pristine, rippled PEN and TCPS control samples (Figure 6). One can observe that the highest values of absorbance were achieved at control samples, proving that TCPS is a material with optimized properties for the cultivation of eukaryotic cells in vitro (cultivation standard) [23]. It could be, therefore, assumed 


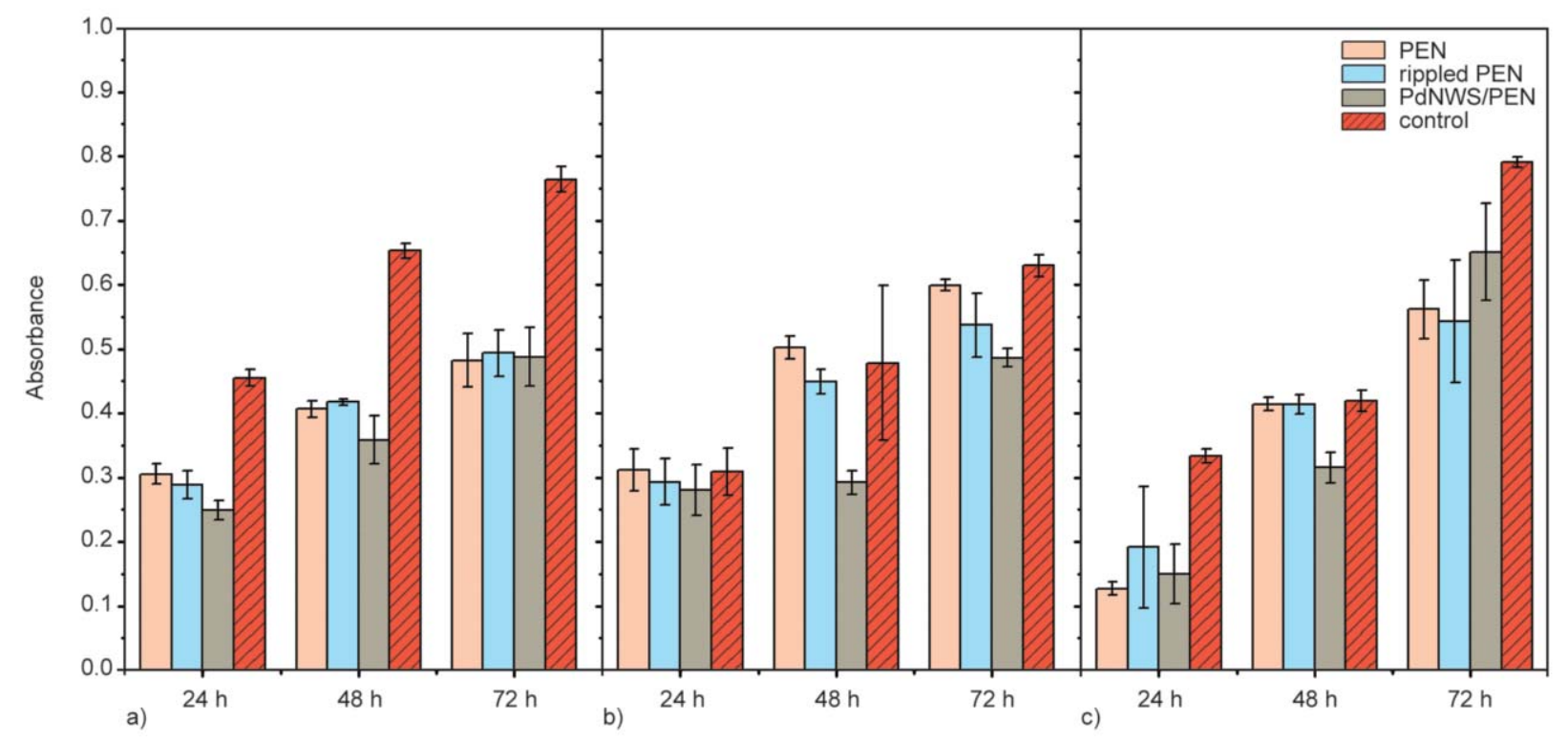

Figure 6. Cytotoxicity of pristine, rippled PEN and PEN/Pd NWs samples after 24, 48 and 72 h of incubation with L929 (a), NIH 3 T3 (b), and RAW 264.7 (c) cells. The absorbance corresponds to relative cell viability.

that pristine and rippled PEN will not reach the same absorbance values. Generally, the growth of the cell lines was not influenced by relatively high surface roughness of rippled PEN as evidenced by the results for pristine and rippled PEN, which exhibited corresponding absorbance values. Surprisingly, when compared absorbances of PEN/Pd samples to pristine and rippled ones, one can see that they did not differ significantly. The absorbance of PEN/PdNWs samples was only negligibly decreased compared to pristine and laser-modified ones, suggesting that Pd exhibited very low cytotoxicity. Unlike PdNWs samples, which we found quite cell-conform, literature refers about noticeable cytotoxic effect of AgNWs $[9,10]$. Even if the comparative study addressing the biological effects of both metal nanowires $(\mathrm{Pd}, \mathrm{Ag})$ at once does not exist, results of our study, conducted under the same experimental conditions as in work [10], have this ambition. Ag cytotoxicity was determined higher than $50 \%$ of TCPS and increasing with cultivation time [10], while our findings pointing to generally featureless cytotoxicity of PdNWs predetermining them to be a safer alternative to AgNWs in its medical applications.

\section{Conclusions}

We have effectively rippled polyethylene naphthalate by excimer laser and prepared palladium nanowire arrays on its surface by vacuum evaporation technique. Due to experimental set-up of vacuum evaporation, surface analyses revealed that PdNWs were formed preferably from one side of underlying ripples, resulting in well-separated NWs. Combination of XPS, FIB-SEM and AFM analyses proved formation of fully separated PdNWs with pretty homogenous distribution over the rippled PEN. Mutual evaluation of antibacterial and cytotoxicity testing, especially pronounce antibacterial efficacy in combination with featureless cytotoxic effect, predetermine PdNWs as a suitable candidate in medical device coatings.

\section{Acknowledgements}

Financial support from Ministry of Health under the project no. 15-32497A and GACR project no. 17-10907S are gratefully acknowledged.

\section{References}

[1] Fernández-García M., Martínez-Arias A., Salamanca L. N., Coronado J. M., Anderson J. A., Conesa J. C., Soria $\mathrm{J}$. : Influence of ceria on $\mathrm{Pd}$ activity for the $\mathrm{CO}+\mathrm{O}_{2}$ reaction. Journal of Catalysis, 187, 474-485 (1999). https://doi.org/10.1006/jcat.1999.2624

[2] Reetz M. T., Westermann E.: Phosphane free palladium-catalyzed coupling reactions: The decisive role of Pd nanoparticles. Angewandte Chemie International Edition, 39, 165-168 (2000).

https://doi.org/10.1002/(SICI)15213773(20000103)39:1<165::AID-ANIE165>3.0.CO;2-B

[3] Tobiška P., Hugon O., Trouillet A., Gagnaire H.: An integrated optic hydrogen sensor based on SPR on palladium. Sensors and Actuators B: Chemical, 74, 168-172 (2001).

https://doi.org/10.1016/S0925-4005(00)00728-0 
[4] Xiong Y., Xia Y.: Shape controlled synthesis of metal nanostructures: The case of palladium. Advanced Materials, 19, 3385-3391 (2007).

https://doi.org/10.1002/adma.200701301

[5] Xu Y., Wen W., Wu J-M.: Titania nanowires functionalized polyester fabrics with enhanced photocatalytic and antibacterial performances. Journal of Hazardous Materials, 343, 285-297 (2018).

https://doi.org/10.1016/j.jhazmat.2017.09.044

[6] Dhanasekar M., Jenefer V., Nambiar R. B., Babu S. G., Selvam S. P., Neppolian B., Bhat S. V.: Ambient light antimicrobial activity of reduced graphene oxide supported metal doped $\mathrm{TiO}_{2}$ nanoparticles and their PVA based polymer nanocomposite films. Materials Research Bulletin, 97, 238-243 (2018).

https://doi.org/10.1016/j.materresbull.2017.08.056

[7] Faustini M., Boissière C., Nicole L., Grosso D.: From chemical solutions to inorganic nanostructured materials: A journey into evaporation-driven processes. Chemistry of Materials, 26, 709-723 (2014).

https://doi.org/10.1021/cm402132y

[8] Moro T., Takatori Y., Ishihara K., Konno T., Takigawa Y., Matsushita T., Chung U-I., Nakamura K., Kawaguchi H.: Surface grafting of artificial joints with a biocompatible polymer for preventing periprosthetic osteolysis. Nature Materials, 3, 829-836 (2004).

https://doi.org/10.1038/nmat1233

[9] Kim M. J., Shin S.: Toxic effects of silver nanoparticles and nanowires on erythrocyte rheology. Food and Chemical Toxicology, 67, 80-86 (2014).

https://doi.org/10.1016/j.fct.2014.02.006

[10] Polívková M., Štrublová V., Hubáček T., Rimpelová S., Švorčík V., Siegel J.: Surface characterization and antibacterial response of silver nanowire arrays supported on laser-treated polyethylene naphthalate. Materials Science and Engineering C, 72, 512-518 (2017). https://doi.org/10.1016/j.msec.2016.11.072

[11] Siegel J., Polívková M., Staszek M., Kolářová K., Rimpelová S., Švorčík V.: Nanostructured silver coatings on polyimide and their antibacterial response. Materials Letters, 145, 87-90 (2015).

https://doi.org/10.1016/j.matlet.2015.01.050

[12] Polívková M., Siegel J., Rimpelová S., Hubáček T., Kolská Z., Švorčík V.: Cytotoxicity of Pd nanostructures supported on PEN: Influence of sterilization on $\mathrm{Pd} / \mathrm{PEN}$ interface. Materials Science and Engineering: C, 70, 479-486 (2017).

https://doi.org/10.1016/j.msec.2016.09.032

[13] Belardini A., Larciprete M., Centini M., Fazio E., Sibilia C., Bertolotti M., Toma A., Chiappe D., de Mongeot F. B.: Tailored second harmonic generation from self-organized metal nano-wires arrays. Optics Express, 17, 3603-3609 (2009).

https://doi.org/10.1364/OE.17.003603
[14] Toma A., Chiappe D., Massabó D., Boragno C., de Mongeot F. B.: Self-organized metal nanowire arrays with tunable optical anisotropy. Applied Physics Letters, 93, 163104/1-163104/3 (2008).

https://doi.org/10.1063/1.3002319

[15] Bolle M., Lazare S.: Large scale excimer laser production of submicron periodic structures on polymer surfaces. Applied Surface Science, 69, 31-37 (1993). https://doi.org/10.1016/0169-4332(93)90478-T

[16] Slepička P., Neděla O., Siegel J., Krajcar R., Kolská Z., Švorčík V.: Ripple polystyrene nano-pattern induced by KrF laser. Express Polymer Letters, 8, 459-466 (2014). https://doi.org/10.3144/expresspolymlett.2014.50

[17] Lewis K. B., Ratner B. D.: Observation of surface rearrangement of polymers using ESCA. Journal of Colloid and Interface Science, 159, 77-85 (1993).

https://doi.org/10.1006/jcis.1993.1299

[18] Slepička P., Michaljaničová I., Švorčík V.: Controlled biopolymer roughness induced by plasma and excimer laser treatment. Express Polymer Letters, 7, 950--958 (2013).

https://doi.org/10.3144/expresspolymlett.2013.92

[19] Polívková M., Valová M., Siegel J., Rimpelová S., Hubáček T., Lyutakov O., Švorčík V.: Antibacterial properties of palladium nanostructures sputtered on polyethylene naphthalate. RSC Advances, 5, 73767-73774 (2015). https://doi.org/10.1039/C5RA09297C

[20] Bachinger T., Mårtensson P., Mandenius C-F.: Estimation of biomass and specific growth rate in a recombinant Escherichia coli batch cultivation process using a chemical multisensor array. Journal of Biotechnology, 60, 55-66 (1998). https://doi.org/10.1016/S0168-1656(97)00187-9

[21] Slepička P., Siegel J., Lyutakov O., Slepičková Kasálková N., Kolská Z., Bačáková L., Švorčík V.: Polymer nanostructures for bioapplications induced by laser treatment. Biotechnology Advances, 36, 839-855 (2017). https://doi.org/10.1016/j.biotechadv.2017.12.011

[22] Tran N., Tran P. A.: Nanomaterial-based treatments for medical device-associated infections. ChemPhysChem, 13, 2481-2494 (2012). https://doi.org/10.1002/cphc.201200091

[23] Pešáková V., Kubies D., Hulejová H., Himmlová L.: The influence of implant surface properties on cell adhesion and proliferation. Journal of Materials Science: Materials in Medicine, 18, 465-473 (2007). https://doi.org/10.1007/s10856-007-2006-0 